\section{Prevalência em crianças de fatores de risco para as doenças cardiovasculares}

\author{
Childhood prevalence of cardiovascular risk factors
}

\begin{abstract}
Cardiovascular risk factors were investigated in 356 children 5 to 9 years of age who were treated at a primary care center located in a low-income area in Greater Metropolitan Rio de Janeiro, Brazil. Lipid profile, nutritional status, food intake, and lifestyle were evaluated. $10.7 \%$ of the children were overweight, $68.4 \%$ had some type of dyslipidemia, and $18.6 \%$ showed high LDL-c. To describe the food intake pattern, the answers to the qualitative food questionnaire were submitted to multivariate cluster analysis, producing six basic groups: traditional Brazilian cooking; "modern" food (including diet and light products); fried food; sweets and soft drinks (mixed with other groups); and other poorly defined groups. The high prevalence of cardiovascular risk factors (beginning in childhood) and the evidence of inadequate dietary habits indicate that a preventive family-focused strategy is needed to change the dietary pattern of low-income groups towards healthier eating.
\end{abstract}

Food Habits; Dyslipidemias; Obesity; Cardiovascular Diseases; Child
Sueli Rosa Gama 1

Marilia Sá Carvalho 1

Célia Regina Moutinho de Miranda Chaves 2

\section{Introdução}

As doenças cardiovasculares têm a obesidade e dislipidemias como principais fatores de risco, que, embora possam se iniciar precocemente 1 são então pouco valorizados clinicamente. A obesidade na infância, o mais importante fator de risco conhecido para as doenças cardiovasculares na vida adulta, apresenta crescente prevalência, associada às mudanças no modo de viver, particularmente o sedentarismo e maior consumo de gorduras e açúcares ${ }^{1,2}$. As alterações do perfil lipídico, com início na mesma faixa etária, ocorrem silenciosamente, sendo a lesão aterosclerótica somente diagnosticada na idade adulta 3 . Pouco se conhece em relação à prevalência de dislipidemias em crianças em idade escolar.

Neste estudo estimamos indicadores do perfil lipídico, estado nutricional, pressão arterial e atividade física em escolares atendidos em unidade básica de saúde localizada em área de baixa renda da Região Metropolitana do Rio de Janeiro, Brasil. Além disso, analisamos os padrões de consumo alimentar, para melhor compreender hábitos alimentares da população de crianças de regiões empobrecidas.

\section{Métodos}

Foram avaliadas 356 crianças (amostra aleatória sistemática), com base nas fichas das crianças 
que compareceram por qualquer motivo, exceto consulta de nutrição, ao Centro de Saúde Escola Germano Sinval Faria, Escola Nacional de Saúde Pública Sergio Arouca, Fundação Oswaldo Cruz (CSEGSF/ENSP/FIOCRUZ), residentes no Complexo de Manguinhos, região de favelas no entorno da FIOCRUZ, entre fevereiro e julho de 2004. O questionário foi adaptado a partir de instrumento utilizado em pesquisa anterior 4 .

A avaliação nutricional utilizou o índice de peso/altura (sobrepeso $>2$ escores z) 5 e para o consumo, questionário de freqüência alimentar qualitativo adaptado 6 .

A avaliação bioquímica foi realizada no Instituto de Pesquisa Evandro Chagas (IPEC/ FIOCRUZ), sendo a coleta de sangue após jejum de 12 horas. Os exames foram realizados pelo método enzimático e os valores de normalidade utilizados foram referidos da I Diretriz de Prevenção da Aterosclerose na Infância e na Adolescência 7: colesterol-c < 170mg/dl; HDL-c > 45mg/dl; LDL$\mathrm{c}<130 \mathrm{mg} / \mathrm{dl}$; triglicerídeo $<130 \mathrm{mg} / \mathrm{dl}$. O cálculo do LDL-c foi obtido pela fórmula de Friedewald \& Fredrickson (LDL-c = Col-T-[HDL+TG/5]). Qualquer alteração do lipidograma foi considerada dislipidemia. As atividades físicas referem-se à freqüência diária dos últimos seis meses.

O padrão de consumo foi caracterizado por análise de cluster da freqüência informada de consumo de cada alimento. As distâncias entre observações basearam-se em matriz de dissimilaridade apropriada para variáveis categóricas, utilizando métrica euclidiana. O critério de agrupamento baseia-se na minimização da soma das distâncias entre cada observação e o centróide do grupo. O número de agrupamentos escolhidos baseou-se na comparação do ajuste obtido para 4 a 8 grupos 8 .

A pesquisa foi aprovada em comitê de ética institucional e analisada no programa $\mathrm{R}$ (The R Foundation for Statistical Computing, Viena, Áustria; http://www.r-project.org).

\section{Resultados}

Cerca de $20 \%$ das crianças moravam só com a mãe, $7 \%$ sem pais. A renda mensal per capita de $45 \%$ foi inferior a $1 / 3$ do salário mínimo, embora $86,7 \%$ tivessem moradia própria, ainda que sem título de propriedade e $71 \%$, telefone. O ensino fundamental incompleto foi relatado por $35,8 \%$ das mães e $2,5 \%$ de crianças estavam fora da escola (Tabela 1).

Encontrou-se prevalência de $10,7 \%$ de sobrepeso e $43 \%$ das crianças ficavam em atividades sedentárias mais de 3 horas por dia (TV ou jogos eletrônicos). O perfil lipídico foi avaliado em so- mente 231 crianças. O não retorno foi mais freqüente em crianças mais pobres, sem telefone e com família desestruturada, sem presença materna. Considerando qualquer alteração do perfil lipídico - colesterol total ou frações - 68,4\% das crianças apresentaram alguma dislipidemia, sendo que $18,6 \%$ tinham aumento da lipoproteína LDL-c, 35\% alteração do HDL-c e 3,5\% do triglicerídeo. Entre os comportamentos familiares de risco para doenças cardiovasculares sobressai o consumo de álcool e fumo, com metade das famílias apresentando pelo menos um destes comportamentos (Tabela 2).

A Figura 1 apresenta a posição dos alimentos consumidos em um par de eixos construído pelos dois primeiros vetores da análise de componentes principais, responsáveis por aproximadamente $60 \%$ da variabilidade total das crianças. Os agrupamentos podem ser caracterizados da seguinte forma:

- Grupo 1: arroz, feijão, óleos, pão francês e leite integral, caracterizando alimentação tradicional;

- Grupo 2: bebidas industrializadas, biscoitos, balas e frutas. É o grupo com maior variabilidade interna, pois as crianças tanto consomem estes alimentos como os de outros grupos. Há predominância de açúcares simples, sem incluir alimentos usuais de refeições principais;

- Grupo 3: frituras e carboidratos preparados, com predominância de gorduras saturadas e trans;

- Grupo 4: disperso, parcialmente lanche (pãode-forma, hambúrguer, maionese e café). Porém, neste grupo encontram-se legumes e verduras;

- Grupo 5: todos os queijos, doces cremosos e carnes. Grupo rico em gordura saturada;

- Grupo 6: produtos dietéticos, bebidas alcoólicas e naturais, mel/melado. É o grupo com menor dispersão, porque todos estes alimentos aparecem nas mesmas crianças.

\section{Discussão}

O perfil familiar deste estudo aponta para uma estrutura fora do padrão nuclear, freqüentemente encontrado em áreas metropolitanas empobrecidas ${ }^{9}$. As posses de moradia própria e telefone foram referidas em mais da metade do grupo, apesar da renda per capita ser 1/3 do salário mínimo. Esta contradição reflete a dificuldade para estimar a real renda familiar, onde o trabalho informal predomina 10 .

Foi importante a presença dos fatores de risco para doenças cardiovasculares na amostra, com predominância da dislipidemia e do sobrepeso. Este último, com prevalência maior do 
Características sócio-econômicas e demográficas de crianças de 5 a 9 anos atendidas no Centro de Saúde Escola Germano Sinval Faria, Escola Nacional de Saúde Pública Sergio Arouca, Fundação Oswaldo Cruz. Rio de Janeiro, Brasil, 2004.

\begin{tabular}{|c|c|c|}
\hline Indicadores & $\mathrm{n}$ & $\%$ \\
\hline \multicolumn{3}{|l|}{ Constituição familiar } \\
\hline Pai e mãe & 187 & 52,5 \\
\hline Mãe e avó & 23 & 6,4 \\
\hline Só mãe & 68 & 19,1 \\
\hline Mãe e padrasto & 43 & 12,0 \\
\hline Sem pai e mãe & 25 & 7,0 \\
\hline Outros & 10 & 3,0 \\
\hline \multicolumn{3}{|c|}{ Número de pessoas no domicílio } \\
\hline$\leq 4$ & 173 & 48,6 \\
\hline$>4$ & 183 & 51,4 \\
\hline Moradia própria & 309 & 86,7 \\
\hline Telefone no domicílio & 253 & 71,0 \\
\hline Carro & 37 & 10,3 \\
\hline \multicolumn{3}{|c|}{ Renda mensal familiar per capita (Reais) } \\
\hline$<100$ & 158 & 44,4 \\
\hline $101-200$ & 132 & 37,1 \\
\hline$>200$ & 66 & 18,5 \\
\hline \multicolumn{3}{|l|}{ Escolaridade da mãe } \\
\hline Não-alfabetizada & 10 & 2,8 \\
\hline Fundamental incompleto & 126 & 35,5 \\
\hline Fundamental completo & 148 & 42,0 \\
\hline Médio & 66 & 18,8 \\
\hline Superior & 2 & 0,6 \\
\hline \multicolumn{3}{|l|}{ Tipo de escola da criança } \\
\hline Pública & 320 & 90,0 \\
\hline Particular & 27 & 7,5 \\
\hline Fora da escola & 9 & 2,5 \\
\hline
\end{tabular}

que a do estudo de Anjos et al. 11 e confirmando o aumento de sobrepeso infantil 12, aponta o aumento do sedentarismo além de inadequado consumo alimentar. A manutenção do peso saudável é importante durante a infância, pois a obesidade adquirida tende a persistir na vida adulta, acompanhada de hipertensão e diabetes, notórios fatores de risco para doenças cardiovasculares. Inicialmente o estudo encontrou prevalência elevada de dislipidemia $(64,5 \%)$ de acordo com a referência do NCEP 13 .

Avaliando os pontos de corte da nova diretriz para prevenção da aterosclerose na infância/adolescência 7, constatou-se ainda maior prevalência estimada $(68,4 \%)$ 3,14. Este resultado, observado mesmo em crianças eutróficas, indica a necessidade da investigação do perfil lipídico, mesmo em crianças eutróficas. Valor elevado de lipoproteína LDL-c favorece o aparecimento da doença arterial coronariana, e conjuntamente com o aumento de triglicerídeo, a síndrome plurimetabólica. Diminuição da lipoproteína HDL-c e aumento do LDL-c e do triglicerídeo elevam em vinte vezes o desenvolvimento da enfermidade coronariana 3 . A lipoproteína HDL-c protege contra as doenças cardiovasculares.

O não comparecimento de crianças para a coleta de sangue, a ser realizada em jejum, em dia posterior à entrevista, é esperado neste tipo de estudo, realizado na rotina dos serviços de saúde. Ressalte-se que as crianças que não vieram fazer os exames tinham renda menor e famílias menos estruturadas. Entretanto, se todas as crianças que não compareceram à coleta de sangue tivessem valores normais, a prevalência de dislipidemia cairia para $44 \%$, ainda extremamente elevada na faixa etária. Repetindo a classificação do padrão alimentar somente sobre as crianças que não compareceram ao exame, obtivemos resultado semelhante, particularmente nos grupos extre- 
Estado nutricional e prevalência de fatores de risco para doenças cardiovasculares em crianças de 5 a 9 anos atendidas no Centro de Saúde Escola Germano Sinval Faria, Escola Nacional de Saúde Pública Sergio Arouca, Fundação Oswaldo Cruz. Rio de Janeiro, Brasil, 2004.

\begin{tabular}{|c|c|c|}
\hline Indicadores & $\mathbf{n}$ & $\%$ \\
\hline \multicolumn{3}{|l|}{ Sobrepeso } \\
\hline Não & 318 & 89,3 \\
\hline Sim & 38 & 10,7 \\
\hline \multicolumn{3}{|c|}{ Atividades sedentárias (minutos/dia) } \\
\hline Até 60 & 99 & 27,8 \\
\hline $60-180$ & 104 & 29,2 \\
\hline $180-350$ & 87 & 24,5 \\
\hline 350 ou + & 66 & 18,5 \\
\hline \multicolumn{3}{|c|}{ Atividades físicas (minutos/dia) } \\
\hline Até 60 & 136 & 38,2 \\
\hline $60-180$ & 104 & 29,3 \\
\hline 180 ou + & 116 & 32,5 \\
\hline \multicolumn{3}{|c|}{ Uso de cigarro na presença da criança } \\
\hline Sim & 204 & 57,3 \\
\hline Não & 152 & 42,7 \\
\hline \multicolumn{3}{|c|}{ Consumo de álcool em pessoas convivendo com a criança } \\
\hline Sim & 173 & 48,5 \\
\hline Não & 183 & 51,5 \\
\hline \multicolumn{3}{|c|}{ Lipídeos séricos $(n=231)$} \\
\hline \multicolumn{3}{|l|}{ Colesterol-T } \\
\hline Normal & 130 & 56,2 \\
\hline Alterado & 101 & 43,8 \\
\hline \multicolumn{3}{|l|}{ LDL-c } \\
\hline Normal & 188 & 81,4 \\
\hline Alterado & 43 & 18,6 \\
\hline \multicolumn{3}{|l|}{ HDL-C } \\
\hline Normal & 150 & 64,9 \\
\hline Alterado & 81 & 35,1 \\
\hline \multicolumn{3}{|l|}{ Triglicerídeo } \\
\hline Normal & 223 & 96,5 \\
\hline Alterado & 8 & 3,5 \\
\hline \multicolumn{3}{|c|}{ Hiperlipidemia mista } \\
\hline Alterado & 4 & 1,7 \\
\hline \multicolumn{3}{|c|}{ HDL/Triglicerídeo/LDL alterados } \\
\hline Alterado & 2 & 1,0 \\
\hline \multicolumn{3}{|c|}{ Alguma dislipidemia } \\
\hline Alterado & 158 & 68,4 \\
\hline
\end{tabular}

mos (1 e 6), ainda que com maior dispersão nos demais grupos. Assim, dado o efeito do perfil de consumo alimentar sobre os lipídeos séricos, a prevalência deve estar acima deste percentual mínimo.

O sedentarismo observado nas crianças indica a recomendação de prática regular de atividade física, a partir de seis anos, conforme diretriz do Healthy People 2000 (http://www.cdc.gov/ youthcampaign; acessado em 09/Dez/2006). O elevado consumo domiciliar de cigarro/álcool reforça a necessidade de abordagem integrada da família.

Na caracterização do consumo alimentar, o grupo de alimentos tradicionais, com perfil pouco disperso, não tem mistura freqüente com os demais alimentos, reforçando os aspectos saudáveis da cultura tradicional brasileira 15 . O 
Agrupamentos alimentares baseados na freqüência de consumo das 356 crianças estudadas, utilizando método de classificação com seis grupos. Centro de Saúde Escola Germano Sinval Faria, Escola Nacional de Saúde Pública Sergio Arouca, Fundação Oswaldo Cruz. Rio de Janeiro, Brasil, 2004.

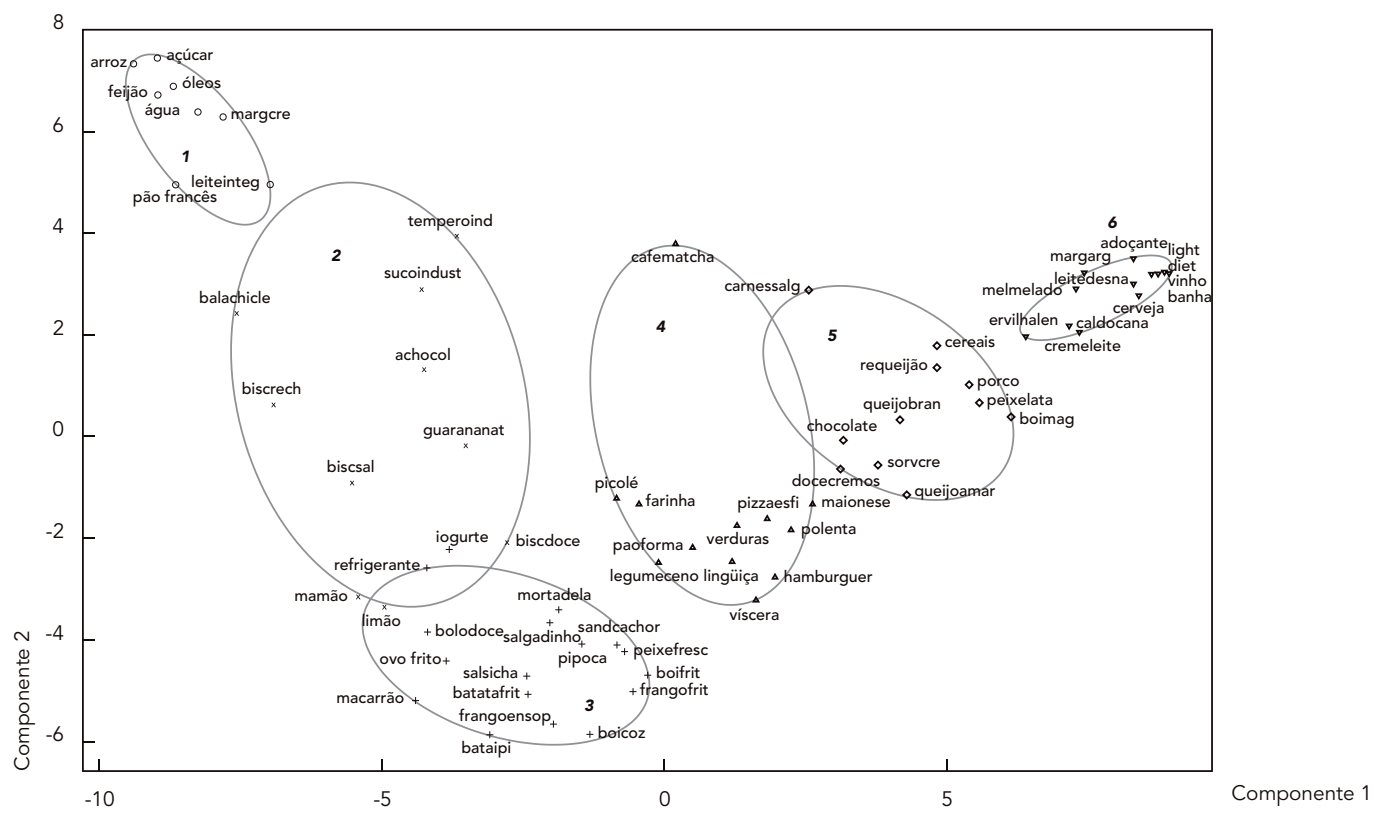

achocol: achocolatado; balachicle: bala e chiclete; bataipi: batata e aipim; batatafrit: batata frita; biscdoce: biscoito doce; biscrech: biscoito recheado; biscsal: biscoito salgado; boicoz: carne de boi cozida; boifrit: carne de boi frita; boimag: carne de boi magra; bolodoce: bolo e doce; cafematcha: café, mate e chá; caldocana: caldo de cana; carnessalg: carnes salgadas; cremeleite: creme de leite; diet: alimentos diet; docecremos: doce cremoso; ervilhalen: ervilha e lentilha; frangoensop: frango ensopado; frangofrit: carne de frango frita; guarananat: guaraná natural; legumeceno: legumes e cenoura; leitedesna: leite desnatado; leiteinteg: leite integral; light: alimentos light; margag: margarina; margcre: margarina cremosa; melmelado: mel e melado; paoforma: pão de forma; peixefresc: peixe fresco; peixelata: peixe em lata; pizzaesfi: pizza e esfirra; porco: carne de porco; queijoamar: queijo amarelo; queijobran: queijo branco; sandcachor: cachorro-quente (sanduíche); sorvcre: sorvete de creme; sucoindust: suco industrializado; temperoind: temperos industrializados.

outro grupo muito bem destacado - dos alimentos dietéticos - poderia ser definido como refletindo a mídia, vendedora de produtos pseudo"saudáveis", que podem levar aos agravos nutricionais. Vale ressaltar que neste grupo inclui-se comportamento perigoso entre as crianças, particularmente, ingestão de bebidas alcoólicas.

Já o grupo caracterizado por bebidas industrializadas, com predominância de açúcares simples mostra comportamento que predispõe ao sobrepeso.
Este estudo aponta a necessidade de trabalhar na prevenção das doenças cardiovasculares desde a infância, integradamente aos programas de saúde da família. Os perfis de consumo alimentar identificados podem contribuir para desenhar estratégias de abordagem desta questão, resgatando aspectos saudáveis da nutrição na população de baixa renda, contrapondo à penetração da indústria alimentícia. 


\section{Resumo}

Investigou-se a presença de fatores de risco para doenças cardiovasculares em estudo transversal em 356 crianças de 5 a 9 anos, atendidas em unidade básica de saúde de área de baixa renda da Região Metropolitana do Rio de Janeiro, Brasil. Foram avaliados: lipidograma, estado nutricional, hábitos alimentares e aspectos sócio-econômicos. Observaram-se 10,7\% de sobrepeso e 68,4\% com niveis alterados no lipidograma, sendo $18,6 \%$ com LDL-colesterol alto. Para descrever o perfil alimentar as respostas ao questionário qualitativo de ingestão foram submetidas à classificação multivariada, obtendo-se seis grupos, resumidamente definidos como: da cultura tradicional brasileira; moderno (produtos diet $e$ light); frituras; doces e refrescos (misturados com outros grupos); os demais pouco definidos. A alta prevalência dos fatores de risco para as doenças cardiovasculares desde a infância e a evidência de alimentação infantil inadequada indica a necessidade de desenvolver uma estratégia preventiva, procurando atingir toda a família, de forma a alterar os padrões de ingestão de alimentos das populações de baixa renda em direção à comportamentos mais saudáveis.

Hábitos Alimentares; Dislipidemias; Obesidade; Doenças Cardiovasculares; Criança

\section{Colaboradores}

S. R. Gama participou da fase de planejamento, coleta de dados, metodologia, interpretação e discussão dos resultados. C. R. M. Chaves participou da fase de planejamento, metodologia e discussão dos resultados. M. S. Carvalho participou da fase de análise e interpretação dos dados e discussão dos resultados. Todos participaram da elaboração do texto.

\section{Agradecimentos}

A toda a equipe do CSEGSF/ENSP/FIOCRUZ que direta ou indiretamente contribuiu na realização da pesquisa, especialmente ao Dr. Marcos Besserman e Dr. Antônio Sérgio Fonseca, diretores no período da realização da pesquisa. Ao IPEC/FIOCRUZ através da sua equipe do laboratório pela análise dos exames de sangue.

Artigo originado da Dissertação de Mestrado apresentada ao Instituto Fernandes Figueira/FIOCRUZ em 15 de fevereiro de 2005, sob o título Detecção de Fatores de Risco para Doenças Cardiovasculares em Crianças de 5 a 9 Anos de Idade Atendidas em uma Unidade Básica de Saúde.

\section{Referências}

1. Williams CL, Hayman LL, Daniels SR, Robinson TN, Steinberger J, Paridon S, et al. Cardiovascular health in childhood: a statement for health professionals from the Committee on Atherosclerosis, Hypertension and Obesity in the Young (AHOY) of the Council on Cardiovascular Disease in the Young, American Heart Association. Circulation 2002; 106:143-60.

2. Nicholson RN. The effect of cardiovascular health promotion on health behaviors in elementary school children: an integrative review. J Pediatr Nurs 2000; 15:343-55.

3. Berenson GS, Srinivasan SR, Bao W, Newman 3rd WWP, Tracy RE, Wattigney WA. Association between multiple cardiovascular risk factors and atherosclerosis in children and young adults. The Bogalusa Heart Study. N Engl J Med 1998; 338:1650-6.
4. Rodrigues LG. Obesidade infantil: associação de grau de adiposidade com os fatores de risco para doenças cardiovasculares [Dissertação de Mestrado]. Rio de Janeiro. Instituto Fernandes Figueira, Fundação Oswaldo Cruz; 1998.

5. Hamill PV, Drizd TA, Johnson CL, Reed RB, Roche AF. NCHS growth curves for children birth-18 years. United States. Vital Health Stat 11 1977; (165):i-iv, 1-74.

6. Sichiery LR. Epidemiologia da obesidade. Rio de Janeiro: Eduerj; 1998.

7. Giuliano ICB, Caramelli B, Pellanda L, Duncan B, Mattos S, Fonseca FH, editores. I diretriz de prevenção da aterosclerose na infância e na adolescência. Arq Bras Cardiol 2005; 85 Suppl 6:3-36.

8. Kaufman L, Rousseeuw PJ. Finding groups in data: an introduction to cluster analysis. New York: John Wiley and Sons; 1990. 
9. Carvalho IMM, Almeida PH. Família e proteção social. São Paulo Perspec 2003; 17:109-22.

10. Guimarães IB. Participação familiar e trabalhadores informais. http://www.abep.nepo.unicamp.br (acessado em 28/Mai/2006).

11. Anjos LA, Castro IRR, Engstrom EM, Azevedo AM Crescimento e estado nutricional em amostra probabilística de escolares no Município do Rio de Janeiro, 1999. Cad Saúde Pública 2003; 19 Suppl 1: S171-9.

12. Giugliano R, Melo ALP. Diagnóstico de sobrepeso e obesidade em escolares: utilização do índice de massa corporal segundo padrão internacional. J Pediatr 2004; (80):129-34.
13. National Cholesterol Education Program. Report of the Expert Panel on Blood Cholesterol Levels in Children and Adolescents. Bethesda: National Heart Lung and Blood Institute, Public Health Service, U.S. Department of Health and Human Services; 1991. (NIH Publication, 91-2732).

14. Romaldini CC, Issier H, Cardoso AL, Diament J, Forti N. Fatores de risco para aterosclerose em crianças e adolescentes com história familiar de doença arterial coronariana prematura. J Pediatr (Rio de J) 2004; 80:134-40.

15. Sichieri LR, Castro JFG, Moura AS. Fatores associados ao padrão de consumo alimentar da população brasileira urbana. Cad Saúde Pública 2003; 19:44-53.

Recebido em 26/Jun/2006

Versão final reapresentada em 19/Mar/2007

Aprovado em 04/Abr/2007 
